\title{
Algebraic reduction of the Ising model
}

\author{
R.J. Baxter \\ Mathematical Sciences Institute, The Australian National \\ University, Canberra, A.C.T. 0200, Australia, e-mail: none
}

\begin{abstract}
We consider the Ising model on a cylindrical lattice of $L$ columns, with fixed-spin boundary conditions on the top and bottom rows. The spontaneous magnetization can be written in terms of partition functions on this lattice. We show how we can use the Clifford algebra of Kaufman to write these partition functions in terms of $L$ by $L$ determinants, and then further reduce them to $m$ by $m$ determinants, where $m$ is approximately $L / 2$. In this form the results can be compared with those of the Ising case of the superintegrable chiral Potts model. They point to a way of calculating the spontaneous magnetization of that more general model algebraically.
\end{abstract}

KEY WORDS: Statistical mechanics, lattice models, transfer matrices.

\section{Introduction}

Onsager calculated the partition function of the two-dimensional square-lattice Ising model in 1944. [1] He did so by observing that the row-to-row transfer matrices generated a finite Lie algebra. This method was refined by Kaufman in 1949 [2], who showed that the matrices could be expressed in terms of a Clifford algebra. By taking the thermodynamic limit of a large lattice, one obtains the free energy per site.

The calculation of the spontaneous magnetization is much harder than that of the free energy. Onsager first announced his famous result for the spontaneous magnetization at a conference at Cornell University in August 1948 and later at a IUPAP conference in Florence. 3, 4, Onsager never published his derivation - the puzzle was finally solved by Yang in 1952. [5]

Here we wish to re-visit this problem, with a view to seeing if the techniques can be generalized to the $N$-state superintegrable case of the solvable chiral Potts model, which has properties very similar to the Ising model and reduces to it when $N=2$. The author has previously used large-lattice functional relations and analytic methods to obtain the spontaneous magnetization of the general solvable chiral Potts model [6, 7], but it would still be interesting 
to have an algebraic method that may give more insight into the properties of the model on a finite lattice.

In section 2 we define the Ising model on a cylindrical lattice of $L$ columns, with fixed-spin boundary conditions on the top and bottom rows. We show how the magnetization can be expressed, firstly as the ratio of partition functions, and consequently as a matrix element between the two ground-state eigenvectors of the transfer matrix $T$. In section 3 we introduce the hamiltonian $\mathcal{H}$ which commutes with $T$ and define "hamiltonian partition functions" $\tilde{Z}, \widetilde{W}$. These are limiting cases of the usual partition functions and are slightly simpler to work with. This $\tilde{Z}$ corresponds to the usual partition function, $\widetilde{W}$ to the partition function with an extra factor $\sigma_{1}$, where $\sigma_{1}$ is some spin deep inside the lattice.

In section 4 we set up the apparatus of the Clifford algebra and use it to write $\tilde{Z}, \widetilde{W}$ as square roots of $L$ by $L$ determinants. Up to this point our calculation parallels that of Yang. [5] Montroll et al [4] also calculated the spontaneous magnetization using the more combinatorial pfaffian method and Szegö's theorem on Toeplitz matrices. [8] In section 5 we marry Yang and Montroll et al's techniques by using Szegö's theorem to evaluate the appropriate large- $L$ limit of $\tilde{W}$ and hence derive Onsager's famous formula for the spontaneous magnetization.

In section 6 we show how the expressions for $\tilde{Z}, \widetilde{W}$ can be reduced to linear expressions in determinants of dimension $[(L-Q) / 2]$, where $Q=0$ or 1 . Then $\tilde{Z}$ is precisely the hamiltonian limit of the corresponding partition function of the two-state superintegrable chiral Potts model. In section 7 we write the result for $\widetilde{W}$ in terms of two orthogonal matrices $B_{+}, B_{-}$.

In section 8 we comment on whether our result for $\widetilde{W}$ can be generalized to the $N$-state superintegrable chiral Potts model.

\section{The model}

The model is defined on the square lattice $\mathcal{L}$, rotated through $45^{\circ}$, with $M+1$ horizontal rows, each containing $L$ spins, as in Fig. 1.

We impose cylindrical boundary conditions, so that the last column $L$ is followed by the first column 1 . At each site $i$ there is a spin $\sigma_{i}$, taking the values +1 or -1 . The spins in the bottom row are fixed to have value $a= \pm 1$, those in the top row to have value +1 . Adjacent spins $\sigma_{i}, \sigma_{j}$ on southwest to northeast edges (with $i$ below $j$ ) interact with Boltzmann weight $\exp \left(K \sigma_{i} \sigma_{j}\right)$; those on southeast to northwest edges with weight $\exp \left(\bar{K} \sigma_{i} \sigma_{j}\right)$.

An important associated parameter is

$$
k^{\prime}=(\sinh 2 K \sinh 2 \bar{K})^{-1} .
$$

The system is ferromagnetically ordered if $K, \bar{K}$ are positive real and

$$
0<k^{\prime}<1 \text {. }
$$

To align with our notation for the chiral Potts model [6, 7] we are defining the RHS of (2.1) to be $k^{\prime}$, rather than $k$ as in Onsager. [1, eq. 2.1a] 


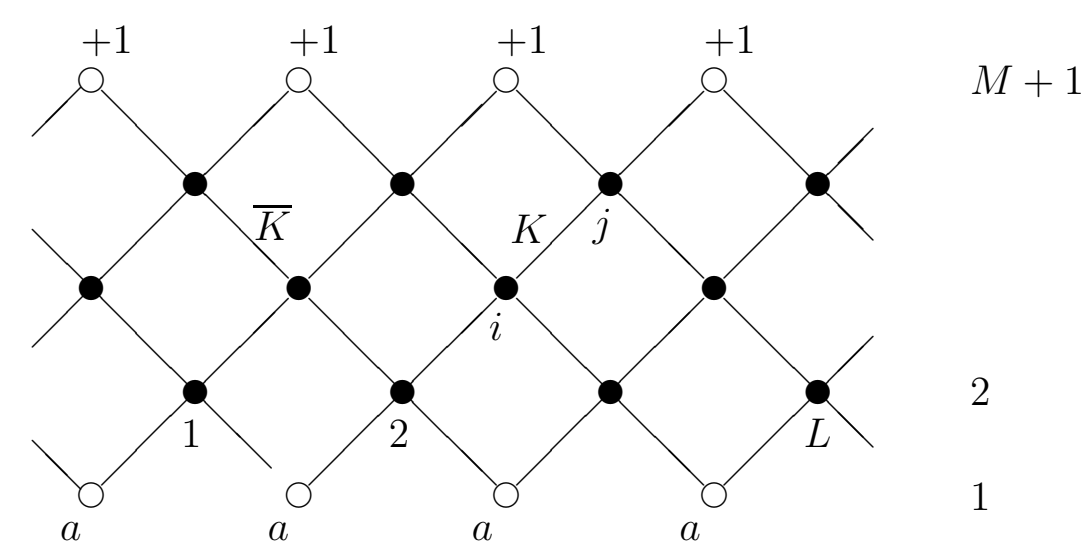

Figure 1: The square lattice $\mathcal{L}$ turned through $45^{\circ}$.

\section{Partition function}

The partition function, which depends on $a$, is

$$
Z_{a}=\sum_{\sigma} \prod_{\langle i, j\rangle} \exp \left(K \sigma_{i} \sigma_{j}\right) \prod_{\langle i, j\rangle} \exp \left(\bar{K} \sigma_{i} \sigma_{j}\right)
$$

the products being over all edges of the two types. The sum is over all values of all the free spins. The partition function can be written as

$$
Z_{a}=u_{a}^{\dagger} T^{M} u_{+},
$$

where $T$ is the row-to-row transfer matrix, with elements

$$
T_{\sigma, \sigma^{\prime}}=\prod_{i=1}^{L} \exp \left(K \sigma_{i} \sigma_{i+1}^{\prime}\right) \exp \left(\bar{K} \sigma_{i} \sigma_{i}^{\prime}\right),
$$

$\sigma$ being the set of all spins $\sigma_{1}, \ldots, \sigma_{L}$ in one row, and $\sigma^{\prime}$ being the set in the row above. Thus $T$ is a $2^{L}$ by $2^{L}$ matrix. The vector $u_{a}$ is of dimension $2^{L}$, with entries

$$
\begin{aligned}
\left(u_{a}\right)_{\sigma} & =1 \text { if } \sigma_{1}=\cdots=\sigma_{L}=a \\
& =0 \text { otherwise. }
\end{aligned}
$$

Two transfer matrices $T, T^{\prime}$, with different values of $K$ and $\bar{K}$, commute provided they have the same value of $k^{\prime}$. [9, section 7.5]

Here $u^{\dagger}$ denoted the transposed conjugate of $u$. However, all our matrices are real, and $T$ commutes with its transpose, so all the eigenvectors we shall discuss are also real: the complex conjugation is unnecessary.

We shall also need the matrices $S_{1}, \ldots, S_{L}, C_{1}, \ldots, C_{L}$, defined by

$$
\left(S_{j}\right)_{\sigma, \sigma^{\prime}}=\sigma_{j} \prod_{n=1}^{L} \delta\left(\sigma_{n}, \sigma_{n}^{\prime}\right)
$$




$$
\left(C_{j}\right)_{\sigma, \sigma^{\prime}}=\delta\left(\sigma_{j},-\sigma_{j}^{\prime}\right) \prod_{n=1}^{L} \delta\left(\sigma_{n}, \sigma_{n}^{\prime}\right),
$$

where the $*$ means that the term $n=j$ is excluded from the product, If $I, S, C$ are the two-by-two matrices

$$
I=\left(\begin{array}{ll}
1 & 0 \\
0 & 1
\end{array}\right), S=\left(\begin{array}{cc}
1 & 0 \\
0 & -1
\end{array}\right), C=\left(\begin{array}{ll}
0 & 1 \\
1 & 0
\end{array}\right),
$$

then we can write $S_{j}, C_{j}$ as

$$
\begin{aligned}
& S_{j}=I \otimes \cdots \otimes I \otimes S \otimes I \otimes \cdots \otimes I \\
& C_{j}=I \otimes \cdots \otimes I \otimes C \otimes I \otimes \cdots \otimes I,
\end{aligned}
$$

the $S, C$ on the RHS being in position $j$ in the sequence of $L$ factors.

\section{Spontaneous magnetization}

Take $a=+1$, so all top and bottom boundary spins are + . Let 0 be a site deep within the lattice. The expectation value of its spin $\sigma_{0}$ is

$$
\mathcal{M}=\left\langle\sigma_{0}\right\rangle=Z_{+}^{-1} \sum_{\sigma} \sigma_{0} \prod_{\langle i, j\rangle} \exp \left(K \sigma_{i} \sigma_{j}\right) \prod_{\langle i, j\rangle} \exp \left(\bar{K} \sigma_{i} \sigma_{j}\right) .
$$

We take the limit when the lattice is infinitely large, so $L, M \rightarrow \infty$, and site 0 is infinitely far from the boundaries.

The $\langle i, j\rangle$ products are unchanged by negating all spins $\sigma_{i}$, so if we imposed toroidal boundary conditions, then it would be true that

$$
\left\langle\sigma_{0}\right\rangle=-\left\langle\sigma_{0}\right\rangle
$$

and this would imply that $\mathcal{M}=0$. At high temperatures $\left(k^{\prime} \geq 1\right)$, this is true also for our fixed-spin boundary conditions when we take the largelattice limit. However, at lower temperaturers $\left(0<k^{\prime}<1\right)$ the system has ferromagnetic long-range order and "remembers" the boundary conditions even in the limit of 0 deep inside a large lattice, so that

$$
0<\mathcal{M}<1 .
$$

If 0 is in column $i$ of row $j+1$, then we can write (2.10) as

$$
\mathcal{M}=u_{+}^{\dagger} T^{j} S_{i} T^{M-j} u_{+} / u_{+}^{\dagger} T^{M} u_{+}
$$

Because of our cylindrical boundary conditions, we can cyclically permute the columns of $\mathcal{L}$, so the RHS must be independent of $i$.

\section{The sub-spaces}

The operator that negates all the spins in a row is

$$
R=C_{1} C_{2} \cdots C_{L}
$$


We can divide the full $2^{L}$-dimensional space into two orthogonal subspaces $\mathcal{V}_{+}, \mathcal{V}_{-}$such that

$$
R v=r v \text { if } v \in \mathcal{V}_{r}, r=+ \text { or }-
$$

The operator $R$ commutes with $T$ and $\mathcal{H}$, so its eigenvectors lie either in $\mathcal{V}_{+}$ or $\mathcal{V}_{-}$. Let $\Lambda_{a}$ be the largest eigenvalue of $T$ in $\mathcal{V}_{a}, \psi_{a}$ the corresponding eigenvector normalized so that $\psi_{a}^{\dagger} \psi_{a}=1$, and define

$$
v_{+}=\left(u_{+}+u_{-}\right) / \sqrt{2}, v_{-}=\left(u_{+}-u_{-}\right) / \sqrt{2} .
$$

Then $T^{j} v_{a} \in \mathcal{V}_{a}$ for all $j$ and

$$
\begin{gathered}
v_{a}^{\dagger} T^{M} v_{b}=0 \text { if } a \neq b, \\
v_{a}^{\dagger} T^{j} S_{i} T^{M-j} v_{b}=0 \text { if } a=b,
\end{gathered}
$$

so (2.13) becomes

$$
\mathcal{M}=\frac{v_{+}^{\dagger} T^{j} S_{i} T^{M-j} v_{-}+v_{-}^{\dagger} T^{j} S_{i} T^{M-j} v_{+}}{v_{+}^{\dagger} T^{M} v_{+}+v_{-}^{\dagger} T^{M} v_{-}} .
$$

\section{Asymptotic degeneracy}

If $j, M-j$ are large we can take $T^{j}=\Lambda_{+}^{j} \psi_{+} \psi_{+}^{\dagger}$ or $\Lambda_{-}^{j} \psi_{-} \psi_{-}^{\dagger}$ (depending on the sub-space in which $T$ is acting). Similarly for $T^{M-j}$ and $T^{M}$. Thus the four matrix elements in (2.19) will be proportional to $\Lambda_{+}^{j} \Lambda_{-}^{M-j}, \Lambda_{-}^{j} \Lambda_{+}^{M-j}$, $\Lambda_{+}^{M}, \Lambda_{-}^{M}$, respectively. However, for $0<k<1, \Lambda_{+}$and $\Lambda_{-}$are asymptotically degenerate, in that for $L$ large

$$
\Lambda_{+} / \Lambda_{-}=1+O\left(\mathrm{e}^{-L \zeta}\right),\left(v_{+}^{\dagger} \psi_{+}\right) /\left(v_{-}^{\dagger} \psi_{-}\right)=1+O\left(\mathrm{e}^{-L \zeta}\right),
$$

choosing the signs of the $\psi_{a}$ appropriately. Here $\zeta$ is independent of $L$ and is a measure of the interfacial tension between the two phases. [9, section 7.10]

That this should be so can be seen from a low-temperature series expansion of the eigenvalues $\Lambda$ and eigenvectors $\psi$ of $T$ (or more conveniently the $\mathcal{H}$ of the next sub-section) in increasing powers of $k^{\prime}$.

One can start from a single zero-temperature configuration in which either all spins are +1 , or all are $-1\left(u_{+}\right.$or $\left.u_{-}\right)$. The expansion then proceeds identically for each choice (going from one choice to the other by negating all spins) until one reaches terms of order $k^{\prime L}$, when one has to consider the opposite state (all spins -1 or all +1 ) from the original. This has the same eigenvalue for $k^{\prime}=0$, so naive eigenvalue perturbation theory fails. Then and only then does one have to decide whether to symmetrize or anti-symmetrize the eigenvector with respect to $R$.

If $\phi_{+}, \phi_{-}$are the corresponding two near-eigenvectors, then up to this order the eigenvalues are the same and

$$
u_{-}^{\dagger} \phi_{+}=u_{+}^{\dagger} \phi_{-}=0 \text {. }
$$

The actual eigenvectors of $T$ are obtained by symmetrizing:

$$
\psi_{+}=\left(\phi_{+}+\phi_{-}\right) / \sqrt{2}, \psi_{-}=\left(\phi_{+}-\phi_{-}\right) / \sqrt{2} .
$$


The relations (2.20) then follow, using (2.16).

Hence in the limit of $j, M-j, L$ all large we can take $\Lambda_{+}=\Lambda_{-}$, and (2.19) becomes

$$
\mathcal{M}=\psi_{+}^{\dagger} S_{i} \psi_{-}
$$

\section{The associated hamiltonian $H$}

Consider the limit when $K$ becomes small and $\bar{K}$ becomes large while $k^{\prime}$ remains fixed. Then to first order in $K$ we obtain from (2.5)

$$
\mathrm{e}^{-L \bar{K}} T=\mathcal{I}-K \mathcal{H}=\mathrm{e}^{-K \mathcal{H}}
$$

where $\mathcal{I}$ is the $2^{L}$-dimensional identity matrix,

$$
\mathcal{H}=\mathcal{H}_{0}+k^{\prime} \mathcal{H}_{1}
$$

where

$$
\mathcal{H}_{0}=-\sum_{i=1}^{L} S_{i} S_{i+1}, \quad \mathcal{H}_{1}=-\sum_{i=1}^{L} C_{i}
$$

and $S_{L+1}=S_{1}$. It follows that this hamiltonian $\mathcal{H}$ commutes with the transfer matrix $T$ and with $R$. Its ground state eigenvector (the one corresponding to the most negative eigenvalue of $\mathcal{H}$, and to the largest eigenvalue of $T$ ) in the subspace $\mathcal{V}_{a}$ will therefore be $\psi_{a}$.

When $k^{\prime}=0$, the hamiltonian reduces to the diagonal matrix $\mathcal{H}_{0}$ and we see that the ground state eigenvectors are indeed $u_{+}$and $u_{-}$, both with eigenvalue $-L$. it is convenient to define the closely related matrix

$$
\mathcal{J}=\mathcal{H}_{0}+L \mathcal{I}=\sum_{i=1}^{L}\left(\mathcal{I}-S_{i} S_{i+1}\right)
$$

This has minimum eigenvalue 0 , the corresponding eigenvectors being $v_{+}$and $v_{-}$.

\section{Hamiltonian partition functions}

We could continue to look at the four matrix elements in (2.19), or attempt to evaluate directly the result (2.23). We prefer to follow an intermediate path and to consider the expressions

$$
\begin{gathered}
\tilde{Z}_{+}(\alpha)=v_{+}^{\dagger} \mathrm{e}^{-\alpha \mathcal{H}} v_{+}, \tilde{Z}_{-}(\beta)=v_{-}^{\dagger} \mathrm{e}^{-\beta \mathcal{H}} v_{-}, \\
\widetilde{W}(\alpha, \beta, x)=v_{+}^{\dagger} \mathrm{e}^{-\alpha \mathcal{H}} \mathrm{e}^{-\rho \mathcal{J}} S_{1} \mathrm{e}^{-\beta \mathcal{H}} v_{-},
\end{gathered}
$$

where

$$
x=\mathrm{e}^{-4 \rho} .
$$

This corresponds to taking the limits $K \rightarrow 0, j, M-j \rightarrow \infty$ in the matrix elements of (2.19), while keeping $\alpha=j K$ and $\beta=K(M-j)$ fixed. 
The advantage of these expressions is that they are finite-lattice partition functions, but with $T^{j}$ replaced by the rather simpler matrix $\mathrm{e}^{-\alpha \mathcal{H}}$. For definiteness, we have taken the $i$ in (2.19) to be 1 .

We have introduced the factor $\mathrm{e}^{-\rho \mathcal{J}}$ into $\widetilde{W}$ because the elements of the diagonal matrix $\mathcal{J}$ take the values $0,4,8, \ldots, 4[L / 2]$, where $[x]$ is the integer part of $x$. Hence $\widetilde{W}(\alpha, \beta, x)$ is a polynomial in $x$ of degree $[L / 2]$. This naturally manifests itself in the following working and provides a useful check against errors.

If $\rho \rightarrow \infty$, so $x \rightarrow 0$, then

$$
\mathrm{e}^{-\rho \mathcal{J}} S_{1} \rightarrow u_{+} u_{+}^{\dagger}-u_{-} u_{-}^{\dagger}=v_{+} v_{-}^{\dagger}+v_{-} v_{+}^{\dagger},
$$

hence

$$
\widetilde{W}(\alpha, \beta, 0)=\tilde{Z}_{+}(\alpha) \tilde{Z}_{-}(\beta)
$$

Also,

$$
\begin{gathered}
\widetilde{W}(\alpha, 0, x)=\tilde{Z}_{+}(\alpha), \\
\widetilde{W}(0, \beta, x)=\tilde{Z}_{-}(\beta) .
\end{gathered}
$$

When $\alpha$ is large and positive, we can replace $\mathrm{e}^{-\alpha \mathcal{H}}$ in the above definitions by $\mathrm{e}^{-\alpha \Lambda_{+}} \psi_{+} \psi_{+}^{\dagger}$ or by $\mathrm{e}^{-\alpha \Lambda_{-}} \psi_{-} \psi_{-}^{\dagger}$, according to the sub-space in which $\mathcal{H}$ is acting. One can then verify that

$$
\mathcal{M}=\lim _{\alpha, \beta, L \rightarrow \infty} \frac{\widetilde{W}(\alpha, \beta, 1)}{\left[\tilde{Z}_{+}(2 \alpha) \tilde{Z}_{-}(2 \beta)\right]^{1 / 2}} .
$$

We show in the following sections that we can use the techniques of Kaufman[2] and Yang[5] to express the $\tilde{Z}, \widetilde{W}$ expressions as square roots of $L$ by $L$ determinants, and hence evaluate $\mathcal{M}$ by using Szegö's theorem on Toeplitz forms. [8, 4] We further show that the determinants themselves can be expressed as squares of $m$ by $m$ determinants, where $L-2 \leq 2 m \leq L$.

\section{The Clifford algebra}

\section{General remarks}

Set

$$
\begin{gathered}
D_{j}={ }_{1} C_{j} S_{j}={ }_{-1} S_{j} C_{j} \\
\Gamma_{j}=C_{1} C_{2} \cdots C_{j-1} D_{j}, \Gamma_{L+j}=C_{1} C_{2} \cdots C_{j-1} S_{j}
\end{gathered}
$$

for $j=1, \ldots, L$.

These $\Gamma_{1}, \ldots, \Gamma_{2 L}$ anti-commute:

$$
\Gamma_{i} \Gamma_{j}+\Gamma_{j} \Gamma_{i}=2 \delta_{i, j} \mathcal{I}
$$

for $1 \leq i, j \leq 2 L$, so form a Clifford algebra. If $P$ is any orthogonal (not necessarily real) $2 L$ by $2 L$ matrix and

$$
\Gamma_{i}^{*}=\sum_{j=1}^{2 L} P_{i, j} \Gamma_{j},
$$


then it is also true that

$$
\Gamma_{i}^{*} \Gamma_{j}^{*}+\Gamma_{j}^{*} \Gamma_{i}^{*}=2 \delta_{i, j} \mathcal{I}
$$

If $V$ is a $2^{L}$-dimensional matrix such that

$$
V \Gamma_{i} V^{-1}=\sum_{j=1}^{2 L} v_{j, i} \Gamma_{j}
$$

for $i=1, \ldots 2 L$, then we say that the $2 L$ by $2 L$ matrix $\widehat{V}$ with elements $v_{i, j}$ is the representative of $V$. If $V$ is real and symmetric, then $\widehat{V}$ is orthogonal and hermitian.

Such matrices form a group $\mathcal{G}$. 10. If $V, V^{\prime}$ have the property (4.6), with representatives $\widehat{V}, \widehat{V^{\prime}}$, then so does $V V^{\prime}$, and its representative is $\widehat{V} \widehat{V^{\prime}}$. Also, if $\widehat{V}=\widehat{V^{\prime}}$, then

$$
V=c V^{\prime}
$$

where $c$ is a scalar factor. This means that a representative matrix determines its parent to within a scalar factor.

If $\Gamma_{i}, \Gamma_{i}^{*}$ are related by (4.4), then

$$
V \Gamma_{i}^{*} V^{-1}=\sum_{j} v_{j, i}^{*} \Gamma_{j}^{*}
$$

where the $v_{i, j}^{*}$ are the elements of the matrix $P \widehat{V} P^{T}$. Thus mapping $\Gamma_{i}$ to $\Gamma_{i}^{*}$ takes $\widehat{V}$ to $P \widehat{V} P^{T}$. Similary, if a matrix $2^{L}$-dimensional $H$ satisfies

$$
H \Gamma_{i}-\Gamma_{i} H=\left(H, \Gamma_{i}\right)=\sum_{j=1}^{2 L} h_{j, i} \Gamma_{j},
$$

where the $h_{i, j}$ are elements of a matrix $\widehat{H}$, then the same mapping takes $\widehat{H}$ to $P \widehat{H} P^{T}$. We call $\hat{H}$ the $H$-representative of $H$.

Any representative $\widehat{V}$ is orthogonal and any $H$-representative $\widehat{H}$ is antisymmetric. If $V(H)$ is hermitian, then (because the $\Gamma_{j}$ are hermitian), then $\widehat{V}(\widehat{H})$ is also hermitian.

\section{Exponentials of $\mathcal{H}, \widehat{\mathcal{H}}$}

If $\widehat{H}$ is hermitian, then it must be pure imaginary and anti-symmetric. It is diagonalizable by a unitary matrix. Further, its eigenvalues are real and occur in pairs $\lambda$ and $-\lambda$, the corresponding eigenvectors being complex conjugates of one another. Adding and subtracting these eigenvectors, it follows that there is a real orthogonal transformation that takes $\widehat{\mathcal{H}}$ to the form

$$
\mathcal{D}=\left(\begin{array}{cc}
0 & 1 D \\
-1 D & 0
\end{array}\right)
$$

where $D$ is a diagonal matrix.

By making such a transformation and then explicitly considering the twoby-two constituent blocks of $\mathcal{D}$, we find that $\exp (-\alpha \mathcal{H}) \in \mathcal{G}$, so

$$
\text { representative of } \exp (-\alpha \mathcal{H})=\exp (-\alpha \widehat{\mathcal{H}}) \text {. }
$$




\section{Partition functions}

If $V$ is hermitian, we can similarly find an orthogonal transformation (4.4) that takes $\widehat{V}$ to block diagonal form, each block being hermitian and orthogonal, so of the form

$$
\left(\begin{array}{cc}
\cosh 2 \alpha_{j} & 1 \sinh 2 \alpha_{j} \\
-1 \sinh 2 \alpha_{j} & \cosh 2 \alpha_{j}
\end{array}\right)
$$

where $j=1, \ldots, L$. If we arrange the $\Gamma_{j}^{*}$ so that this block is in rows and columns $j, L+j$, it follows that

$$
V=c \exp \left(1 \sum_{j=1}^{L} \alpha_{j} \Gamma_{j}^{*} \Gamma_{L+j}^{*}\right),
$$

where $c$ is some scalar factor.

Each term $\Gamma_{j}^{*} \Gamma_{L+j}^{*}$ commutes with all the other terms, so we have decomposed $V$ into a direct product of two-by-two matrices, the $j$ th such matrix having eigenvalues $\mathrm{e}^{\alpha_{j}}, \mathrm{e}^{-\alpha_{j}}$. Hence

$$
\operatorname{trace} V /(\operatorname{det} V)^{1 / d}=\prod_{j=1}^{L} 2 \cosh \alpha_{j},
$$

where $d=2^{L}$. On the other hand, from (4.11),

$$
\operatorname{det}(I+\widehat{V})=\prod_{J=1}^{L} 4 \cosh ^{2} \alpha_{j},
$$

so we have the identity

$$
\text { trace } V /(\operatorname{det} V)^{1 / d}=[\operatorname{det}(I+\widehat{V})]^{1 / 2} .
$$

Note that the relations (4.10), 4.15) are quite general, being unchanged by the orthogonality transformation (4.4).

Representative of $\exp (-\alpha \mathcal{H})$

From (3.3), (4.2),

$$
\begin{gathered}
\mathcal{H}_{0}={ }_{1} R \Gamma_{L} \Gamma_{L+1}-1 \sum_{j=1}^{L-1} \Gamma_{j} \Gamma_{L+j+1} \\
\mathcal{H}_{1}=1 \sum_{j=1}^{L} \Gamma_{j} \Gamma_{L+j}
\end{gathered}
$$

so we see that $\mathcal{H}_{0}, \mathcal{H}_{1}$ are both quadratic forms in the $\Gamma_{i}$, provided we introduce the spin-reversal operator $R$. We can do this, since in either sub-space $\mathcal{V}_{ \pm}, R$ commutes with $T$ and $\mathcal{H}$, having the value $r=+1$ in $\mathcal{V}_{+}$, and $r=-1$ in $\mathcal{V}_{-}$. 
From now on we take $\mathcal{H}_{0}$ to be defined by (4.16) and $\mathcal{J}$ by (3.4), with $R$ fixed to be either $r=+1$ or $r=-1$. The minimum eigenvalue of $\mathcal{J}$ is still zero, but is now unique, the corresponding eigenvector being either $v_{+}$or $v_{-}$.

To calculate the matrix elements $\tilde{Z}, \widetilde{W}$ using (4.15), we shall need to write each as the trace of an operator. We can do this by introducing the operator $\exp (-\gamma \mathcal{J})$. In the limit $\gamma \rightarrow+\infty$ we have

$$
\mathrm{e}^{-\gamma \mathcal{J}}=v_{r} v_{r}^{\dagger}
$$

It follows that

$$
\begin{gathered}
{\left[\mathcal{H}_{0}, \Gamma_{i}\right]=2 \mathrm{1} \Gamma_{L+i+1}, \quad\left[\mathcal{H}_{0}, \Gamma_{L+i}\right]=-21 \Gamma_{i-1},} \\
{\left[\mathcal{H}_{1}, \Gamma_{i}\right]=-21 \Gamma_{L+i}, \quad\left[\mathcal{H}_{1}, \Gamma_{L+i}\right]=21 \Gamma_{i},}
\end{gathered}
$$

for $i=1, \ldots, L$, provided that on the RHS we take $\Gamma_{2 L+1}=-r \Gamma_{L+1}$ and $\Gamma_{0}=-r \Gamma_{L}$.

We shall need some sparse $L$ by $L$ matrices. Let $0, I$ be the zero and identity $L$ by $L$ matrices, respectively, and let $\mathcal{A}, \mathcal{B}$ be the one-off and twooff-diagonal $L$ by $L$ matrices

$$
\mathcal{A}=\left(\begin{array}{ccccccc}
0 & 1 & 0 & . & . & . & . \\
\cdot & 0 & 1 & 0 & & & \\
\cdot & & & \cdot & & & \\
\cdot & & & & . & & \\
\cdot & & & & 0 & 1 & 0 \\
0 & . & & & 0 & 1 \\
-r & 0 & . & . & . & . & 0
\end{array}\right), \mathcal{B}=\left(\begin{array}{ccccccc}
0 & 0 & 1 & 0 & . & \cdot & . \\
\cdot & 0 & 0 & 1 & 0 & & \\
\cdot & & & & \cdot & & \\
\cdot & & & & & \cdot & \\
0 & & & & 0 & 0 & 1 \\
-r & 0 & & & & 0 & 0 \\
0 & -r & 0 & . & . & . & 0
\end{array}\right) .
$$

Note that

$$
\mathcal{A} \mathcal{A}^{T}=\mathcal{A}^{T} \mathcal{A}=I, \mathcal{B}=A^{2} .
$$

Then from (4.18), the $H$-representatives are

$$
\begin{gathered}
\widehat{\mathcal{H}}_{0}=21\left(\begin{array}{cc}
0 & -\mathcal{A} \\
\mathcal{A}^{T} & 0
\end{array}\right), \\
\widehat{\mathcal{H}}_{1}=21\left(\begin{array}{cc}
0 & I \\
-I & 0
\end{array}\right) .
\end{gathered}
$$

\section{Diagonalization of $\mathcal{H}$}

From (3.2), the $H$-representative of $\mathcal{H}$ is $\widehat{\mathcal{H}}_{0}+k^{\prime} \widehat{\mathcal{H}}_{1}$.

We calculate this matrix. First we define a $2 L$ by $2 L$ matrix $M$ (not to be confused with the $M$ of section 2):

$$
M=\left(\begin{array}{cc}
1 & -1 \mathcal{A} \\
\mathcal{A}^{T} & 1
\end{array}\right)
$$


(writing 1 for $1 I$ and 1 for $I$ ). Then

$$
\begin{gathered}
M^{-1} \widehat{\mathcal{H}}_{0} M=2\left(\begin{array}{cc}
-1 & 0 \\
0 & 1
\end{array}\right), \\
M^{-1} \widehat{\mathcal{H}}_{1} M=\left(\begin{array}{cc}
\mathcal{A}+\mathcal{A}^{T} & I-\mathcal{B} \\
I-\mathcal{B}^{T} & -\mathcal{A}-\mathcal{A}^{T}
\end{array}\right) .
\end{gathered}
$$

Thus $M^{-1} \widehat{\mathcal{H}}_{0} M$ is diagonal.

For $j=1, \ldots, L$, define

$$
\begin{aligned}
\theta_{j} & =\pi(2 j-1) / L & & \text { if } r=+, \\
& =2 \pi j / L & & \text { if } r=-,
\end{aligned}
$$

set

$$
z_{j}=\mathrm{e}^{1 \theta_{j}}
$$

and let $\hat{P}, \hat{Q}$ be the $L$ by $L$ matrices with entries

$$
\hat{P}_{i, j}=z_{j}^{i-1} / \sqrt{L}, \hat{Q}_{i, j}=1 z_{j}^{i-2} / \sqrt{L} .
$$

These matrices are unitary, but not orthogonal. Set

$$
\mathcal{P}=\left(\begin{array}{cc}
\hat{P} & 0 \\
0 & \hat{Q}
\end{array}\right) .
$$

Then

$$
\begin{aligned}
& \tilde{\mathcal{H}}_{0}=\mathcal{P}^{-1} M^{-1} \widehat{\mathcal{H}}_{0} M \mathcal{P}=2\left(\begin{array}{cc}
-1 & 0 \\
0 & 1
\end{array}\right), \\
& \tilde{\mathcal{H}}_{1}=\mathcal{P}^{-1} M^{-1} \widehat{\mathcal{H}}_{1} M \mathcal{P}=2\left(\begin{array}{cc}
\tilde{C} & \tilde{S} \\
\tilde{S} & -\tilde{C}
\end{array}\right),
\end{aligned}
$$

where $\tilde{C}, \tilde{S}$ are diagonal $L$ by $L$ matrices with diagonal entries

$$
\tilde{C}_{j, j}=\cos \theta_{j}, \quad \tilde{S}_{j, j}=\sin \theta_{j} .
$$

Thus $\tilde{\mathcal{H}}=\tilde{\mathcal{H}}_{0}+k^{\prime} \tilde{\mathcal{H}}_{1}$ can be re-arranged as a matrix consisting of $L$ twoby-two diagonal blocks. It is then straightforward to calculate its exponential, giving

$$
\exp (-\alpha \tilde{\mathcal{H}})=\left(\begin{array}{cc}
\tilde{U} & \tilde{V} \\
\tilde{V} & \tilde{T}
\end{array}\right),
$$

where $\tilde{U}=\tilde{U}_{r}(\alpha), \tilde{V}=\tilde{V}_{r}(\alpha), \tilde{T}=\tilde{T}_{r}(\alpha)$ are diagonal matrices with diagonal entries (for $j=1, \ldots, L$ )

$$
\begin{gathered}
\tilde{U}_{j j}=u_{j}(r, \alpha)=\cosh \left(2 \alpha \lambda_{j}\right)+\frac{1-k^{\prime} \cos \theta_{j}}{\lambda_{j}} \sinh \left(2 \alpha \lambda_{j}\right), \\
\tilde{V}_{j j}=v_{j}(r, \alpha)=-\frac{k^{\prime} \sin \theta_{j} \sinh \left(2 \alpha \lambda_{j}\right)}{\lambda_{j}}, \\
\tilde{T}_{j j}=t_{j}(r, \alpha)=\cosh \left(2 \alpha \lambda_{j}\right)-\frac{1-k^{\prime} \cos \theta_{j}}{\lambda_{j}} \sinh \left(2 \alpha \lambda_{j}\right)
\end{gathered}
$$

and

$$
\lambda_{j}=\left(1-2 k^{\prime} \cos \theta_{j}+k^{\prime 2}\right)^{1 / 2} .
$$




\section{Calculation of $\tilde{Z}$}

We want to calculate $\tilde{Z}_{+}(\alpha)$ and $\tilde{Z}_{-}(\beta)$ from (3.5). First consider $\tilde{Z}_{+}(\alpha)$, so take $r=+$ in the above equations. If we are working in the sub-space $\mathcal{V}_{+}$, we can use (4.17) to write (3.5) as

$$
\tilde{Z}_{+}(\alpha)=\operatorname{trace} \mathrm{e}^{-\gamma \mathcal{J}} \mathrm{e}^{-\alpha \mathcal{H}}
$$

in the limit $\gamma \rightarrow+\infty$. Since $\mathcal{H}_{0}$ and $\mathcal{H}_{1}$ are traceless,

$$
\operatorname{det} \mathrm{e}^{-\rho \mathcal{H}_{0}}=\operatorname{det} \mathrm{e}^{-\alpha \mathcal{H}}=1
$$

and from (3.4), $\operatorname{det} \mathrm{e}^{-\gamma \mathcal{J}}=\mathrm{e}^{-L d \gamma}, \widehat{\mathcal{J}}=\widehat{\mathcal{H}}_{0}$. From (4.15) we therefore have

$$
\mathrm{e}^{2 L \gamma} \tilde{Z}_{+}(\alpha)^{2}=\operatorname{det}\left(I+\mathrm{e}^{-\gamma \widehat{\mathcal{H}}_{0}} \mathrm{e}^{-\alpha \widehat{\mathcal{H}}}\right)=\operatorname{det}\left(\mathrm{e}^{\gamma \widehat{\mathcal{H}}_{0}}+\mathrm{e}^{-\alpha \widehat{\mathcal{H}}}\right) .
$$

Using the similarity transformation of (4.28), we can replace $\widehat{\mathcal{H}}_{0}, \widehat{\mathcal{H}}$ in (4.35) by $\tilde{\mathcal{H}}_{0}, \tilde{\mathcal{H}}$, giving

$$
\mathrm{e}^{2 L \gamma} \tilde{Z}_{+}(\alpha)^{2}=\operatorname{det}\left(\begin{array}{cc}
\mathrm{e}^{-2 \gamma} I+\tilde{U} & \tilde{V} \\
\tilde{V} & \mathrm{e}^{2 \gamma} I+\tilde{T}
\end{array}\right) .
$$

In the limit of $\gamma$ large we expect the $\mathrm{e}^{-2 \gamma} I, \tilde{V}, \tilde{T}$ blocks in this determinant to become relatively negligible. The factors involving $\gamma$ then cancel, leaving

$$
\tilde{Z}_{+}(\alpha)^{2}=\operatorname{det} \tilde{U}=\operatorname{det} \tilde{U}_{+}(\alpha)=\prod_{j=1}^{L} u_{j}(+, \alpha) .
$$

Similarly,

$$
\tilde{Z}_{-}(\alpha)^{2}=\operatorname{det} \tilde{U}_{-}(\alpha)=\prod_{j=1}^{L} u_{j}(-, \alpha)
$$

\section{Calculation of $\widetilde{W}$}

The function $\widetilde{W}(\alpha, \beta, x)$ is defined by (3.6). We note that the operator $S_{1}$ takes a vector in $\mathcal{V}_{+}$to one in $\mathcal{V}_{-}$, and vice-versa. In particular,

$$
S_{1} v_{r}=v_{-r}
$$

so

$$
\lim _{\gamma \rightarrow+\infty} \mathrm{e}^{-\gamma \mathcal{J}} S_{1}=v_{-r} v_{r}^{\dagger}
$$

We can use this to write (3.6) as a trace:

$$
\widetilde{W}(\alpha, \beta, x)=\operatorname{trace} \mathrm{e}^{-\gamma \mathcal{J}} S_{1} \mathrm{e}^{-\alpha \mathcal{H}} \mathrm{e}^{-\rho \mathcal{J}} S_{1} \mathrm{e}^{-\beta \mathcal{H}},
$$

in the limit $\gamma \rightarrow+\infty$. We take the factors $\mathrm{e}^{-\gamma \mathcal{J}}, \mathrm{e}^{-\beta \mathcal{H}}$ to be acting in $\mathcal{V}_{-}$, the two other exponential factors to be acting in $\mathcal{V}_{+}$.

The reason we are able to make further progress is that $S_{1}$ also belongs to the group $\mathcal{G}$. In fact $S_{1}=\Gamma_{L+1}$. This is linear in the $\Gamma_{j}$, unlike $\mathcal{H}_{0}$ and $\mathcal{H}_{1}$, which from (4.16) are quadratic. Even so,

$$
S_{1} \Gamma_{j} S_{1}^{-1}=\epsilon_{j} \Gamma_{j}
$$


where $\epsilon_{L+1}=+1$, else $\epsilon_{j}=-1$. The representative of $S_{1}$ is therefore

$$
\widehat{S}_{1}=\left(\begin{array}{cc}
-I & 0 \\
0 & -E
\end{array}\right),
$$

where $E$ is the diagonal matrix

$$
E=\left(\begin{array}{cccccc}
-1 & 0 & \cdot & \cdot & \cdot & \cdot \\
0 & 1 & 0 & & & \\
\cdot & & & \cdot & & \\
\cdot & & & 0 & 1 & 0 \\
\cdot & . & . & \cdot & \cdot & 1
\end{array}\right) .
$$

Remembering that $\widehat{\mathcal{J}}=\widehat{\mathcal{H}}_{0}$, the representative of the matrix whose trace is to be evaluated in (4.40) is therefore the $2 L$ by $2 L$ matrix

$$
\widehat{\mathcal{W}}(\alpha, \beta, x)=\mathrm{e}^{-\gamma \widehat{\mathcal{H}}_{0}} \widehat{S}_{1} \mathrm{e}^{-\alpha \widehat{\mathcal{H}}} \mathrm{e}^{-\rho \widehat{\mathcal{H}}} \widehat{S}_{1} \mathrm{e}^{-\beta \widehat{\mathcal{H}}}
$$

and from (4.15)

$$
\mathrm{e}^{2 L(\gamma+\rho)} \widetilde{W}(\alpha, \beta, x)^{2}=\operatorname{det}[I+\widehat{W}(\alpha, \beta, x)] .
$$

We now use the similarity transformation of (4.28). We have to be careful because $A, M, \hat{P}, \hat{Q}, \mathcal{P}$ depend on $r$. We write them more explicitly as $A_{r}, M_{r}, \hat{P}_{r}, \hat{Q}_{r}, \mathcal{P}_{r}$. Similarly we write the $\theta_{j}, z_{j}$ of (4.25) - (4.27) as $\theta_{r, j}, z_{r, j}$. For the middle two exponential factors we take $r=+$, for the others we take $r=-$. The result is

$$
\tilde{\mathcal{W}}=\mathcal{P}_{-}^{-1} M_{-}^{-1} \widehat{\mathcal{W}} M_{-} \mathcal{P}_{-}=\mathrm{e}^{-\gamma \tilde{\mathcal{H}}_{0}} \tilde{S}_{-} \mathrm{e}^{-\alpha \tilde{\mathcal{H}}} \mathrm{e}^{-\rho \tilde{\mathcal{H}}_{0}} \tilde{S}_{+} \mathrm{e}^{-\beta \tilde{\mathcal{H}}}
$$

where

$$
\tilde{S}_{r}=\mathcal{P}_{r}^{-1} M_{r}^{-1} \widehat{S}_{1} M_{-r} \mathcal{P}_{-r} .
$$

This threatens to become messy, but we find some remarkable simplifications. Firstly,

$$
M_{r} \widehat{S}_{1}=\widehat{S}_{1} M_{-r},
$$

so $M_{r}^{-1} \widehat{S}_{1} M_{-r}=\widehat{S}_{1}$ and (4.47) becomes

$$
\tilde{S}_{r}=\mathcal{P}_{r}^{-1} \widehat{S}_{1} \mathcal{P}_{-r}
$$

The second surprise is that

$$
\hat{Q}_{r}^{-1} E \hat{Q}_{-r}=\hat{P}_{r}^{-1} \hat{P}_{-r}=-F_{r},
$$

where $F_{r}$ is the $L$ by $L$ matrix with entries

$$
F_{i, j}^{(r)}=\frac{2 z_{r, i}}{L\left(z_{-r, j}-z_{r, i}\right)}
$$

satisfying

$$
F_{-} F_{+}=I \text {. }
$$


Hence $\tilde{S}_{r}$ has the comparitively simple block-diagonal form

$$
\tilde{S}_{r}=\left(\begin{array}{cc}
F_{r} & 0 \\
0 & F_{r}
\end{array}\right) .
$$

Let

$$
\tilde{Y}=\tilde{S}_{-} \mathrm{e}^{-\alpha \tilde{\mathcal{H}}} \mathrm{e}^{-\rho \tilde{\mathcal{H}}_{0}} \tilde{S}_{+} \mathrm{e}^{-\beta \tilde{\mathcal{H}}},
$$

then from (4.45) and (4.46)

$$
\mathrm{e}^{2 L(\gamma+\rho)} \widetilde{W}(\alpha, \beta, x)^{2}=\operatorname{det}\left(I+\mathrm{e}^{-\gamma \tilde{\mathcal{H}}_{0}} \tilde{Y}\right)=\operatorname{det}\left(\mathrm{e}^{\gamma \tilde{\mathcal{H}}_{0}} I+\tilde{Y}\right) .
$$

The RHS has the same stucture as that of (4.36), so taking the limit $\gamma \rightarrow+\infty$, we obtain, similarly to (4.37),

$$
\mathrm{e}^{2 L \rho} \widetilde{W}(\alpha, \beta, x)^{2}=\operatorname{det} \tilde{Y}_{11},
$$

where $\tilde{Y}_{11}$ is the top-left $L$ by $L$ block of $\tilde{Y}$. Using (4.28) and (4.30), it follows that

$$
\widetilde{W}(\alpha, \beta, x)^{2}=\operatorname{det}\left[F_{-} \tilde{U}_{+}(\alpha) F_{+} \tilde{U}_{-}(\beta)+x F_{-} \tilde{V}_{+}(\alpha) F_{+} \tilde{V}_{-}(\beta)\right] .
$$

Set, for $r=+$ or - ,

$$
X_{r}(\alpha)=\left[\tilde{U}_{r}(\alpha)\right]^{-1} \tilde{V}_{r}(\alpha)
$$

Then from (4.37) and (4.38), remembering that the $\tilde{U}, \tilde{V}$ matrices are diagonal, so commute, we can write (4.57) as

$$
\widetilde{W}(\alpha, \beta, x)^{2}=\tilde{Z}_{+}(\alpha)^{2} \tilde{Z}_{-}(\beta)^{2} \operatorname{det}\left[I+x F_{+}^{-1} X_{+}(\alpha) F_{+} X_{-}(\beta)\right] .
$$

To summarize thus far, the equations (4.37), (4.38), (4.59) give $\tilde{Z}_{+}(\alpha)$, $\tilde{Z}_{-}(\beta), \widetilde{W}(\alpha, \beta, x)$ in terms of $L$ by $L$ determinants, and these formulae are exact for finite $\alpha, \beta, L$. The spontaneous magnetization $\mathcal{M}$ is then given by (3.11), taking the limit $\alpha, \beta, L \rightarrow+\infty$.

\section{Calculation of $\mathcal{M}$}

So far we have parallelled the method of Yang. [5] We now explicitly calculate $\mathcal{M}$ from the above equations, but we use a method more like that of Montroll, Potts and Ward. [4]

First set $x=1$ and take the limit $\alpha, \beta \rightarrow+\infty$ in (4.37), (4.38), (4.59), using (4.31). We obtain

$$
\mathcal{M}^{2}=\xi_{+} \xi_{-} \operatorname{det}\left[I+F_{+}^{-1} X_{+}(\infty) F_{+} X_{-}(\infty)\right]
$$

where

$$
\xi_{r}=\prod_{j=1}^{L}\left(\frac{\lambda_{r, j}+1-k^{\prime} \cos \theta_{r, j}}{2 \lambda_{r, j}}\right)^{1 / 2}
$$


and $X_{r}(\infty)$ is the diagonal matrix with diagonal entries

$$
\left[X_{r}(\infty)\right]_{j, j}=\frac{-k^{\prime} \sin \theta_{r, j}}{\lambda_{r, j}+1-k^{\prime} \cos \theta_{r, j}} .
$$

From (4.50), $F_{+}=-\hat{P}_{+}^{-1} \hat{P}_{-}$, so if we set

$$
\tilde{X}_{r}=\hat{P}_{r} X_{r}(\infty) \hat{P}_{r}^{-1}
$$

then (5.1) can be written

$$
\mathcal{M}^{2}=\xi_{+} \xi_{-} \operatorname{det}\left[I+\tilde{X}_{+} \tilde{X}_{-}\right] .
$$

We could write this determinant as

$$
\operatorname{det} \tilde{X}_{+} \operatorname{det}\left[\tilde{X}_{+}^{-1}+\tilde{X}_{-}\right]
$$

From the definition (4.26) of $\hat{P}$, the matrices $\tilde{X}_{+}, \tilde{X}_{-}$are anti-cyclic and cyclic, respectively, i.e.

$$
\left[\tilde{X}_{r}\right]_{i, j}=\eta_{i-j}=-r \eta_{L+i-j}
$$

where $\eta$ is defined by this equation. The matrix $\tilde{X}_{+}^{-1}+\tilde{X}_{-}$is therefore Toeplitz, its elements $i, j$ depending only on $i-j$, and one might hope to use Szegö's theorem [4, 8, to evaluate the determinant for large $L$.

Unfortunately this naive approach does not work because for large $L$ the determinant is not dominated by its near-diagonal elements, in particular elements such as $(L, 1),(2, L-3)$ do not tend to zero. However, we can rescue this idea by making a particular bilinear transformation of $\tilde{X}_{+}, \tilde{X}_{-}$.

We set

$$
C_{r}=\left(I-1 \tilde{X}_{r}\right)^{-1}\left(I+1 \tilde{X}_{r}\right)=\hat{P}_{r} c_{r} \hat{P}_{r}^{-1}
$$

where $c_{r}$ is a diagonal matrix with diagonal elements

$$
\left(c_{r}\right)_{j, j}=\frac{\lambda_{r, j}+1-k^{\prime} \exp \left(1 \theta_{r, j}\right)}{\lambda_{r, j}+1-k^{\prime} \exp \left(-1 \theta_{r, j}\right)} .
$$

Solving for $X_{r}$ and substituting into (5.5), we obtain

$$
\mathcal{M}^{2}=\xi_{+}^{\prime} \xi_{-}^{\prime} \operatorname{det}\left[\left(C_{+}+C_{-}\right) / 2\right]
$$

where

$$
\begin{aligned}
\xi_{r}^{\prime} & =\xi_{r} \operatorname{det}\left(I-{ }_{1} \tilde{X}_{r}\right) \\
& =\prod_{j=1}^{L}\left\{\frac{\left[\lambda_{r, j}+1-k^{\prime} \exp \left(-1 \theta_{r, j}\right)\right]^{2}}{2 \lambda_{r, j}\left(\lambda_{r, j}+1-k^{\prime} \cos \theta_{r, j}\right)}\right\}^{1 / 2} .
\end{aligned}
$$

From (4.32),

$$
\lambda_{r, j}^{2}=\left[1-k^{\prime} \exp \left({ }_{1} \theta_{r, j}\right)\right]\left[1-k^{\prime} \exp \left(-1 \theta_{r, j}\right)\right],
$$


from which it follows that

$$
\xi_{r}^{\prime}=\prod_{j=1}^{L}\left[\frac{1-k^{\prime} \exp \left(-1 \theta_{r, j}\right)}{1-k^{\prime} \exp \left(1 \theta_{r, j}\right)}\right]^{1 / 4} .
$$

The $\theta_{r, j}$ are either $\pi$ or $2 \pi$, or occur in pairs $\theta_{r, j}, 2 \pi-\theta_{r, j}$. It follows that

$$
\xi_{+}^{\prime}=\xi_{-}^{\prime}=1
$$

Also, $\left(c_{r}\right)_{j, j}$ simplifies to

$$
\left(c_{r}\right)_{j, j}=\left[\frac{1-k^{\prime} \exp \left(-1 \theta_{r, j}\right)}{1-k^{\prime} \exp \left(1 \theta_{r, j}\right)}\right]^{1 / 2} .
$$

From (4.26) and (5.6), the elements of $C_{r}$ are

$$
\left(C_{r}\right)_{i, j}=\frac{1}{L} \sum_{m=1}^{L}\left(c_{r}\right)_{m, m} z_{r, m}^{i-j},
$$

where $z_{r, m}=\mathrm{e}^{1 \theta_{r, m}}$. The summand depends on $m$ via $\theta_{r, m}$ and is an analytic function of $\theta_{r, m}$ on the real axis, periodic of period $2 \pi$. The $\theta_{r, m}$ are distributed uniformly throughout this period. If $i, j$ are held finite and $L \rightarrow \infty$, it follows that

$$
\left(C_{r}\right)_{i, j}=\frac{1}{2 \pi} \int_{0}^{2 \pi} f(\theta) \mathrm{e}^{1(i-j) \theta} \mathrm{d} \theta
$$

where

$$
f(\theta)=\left[\frac{1-k^{\prime} \exp (-1 \theta)}{1-k^{\prime} \exp (1 \theta)}\right]^{1 / 2} .
$$

This is actually the function $\mathrm{e}^{1 \delta^{*}}$ of Onsager[1, eq. 89] and Montroll et al[4, eq. 42] In their notation $z_{1}=\tanh H^{\prime}, z_{2}^{*}=\tanh H^{*}$ and our $k^{\prime}$ is $k^{\prime}=z_{2}^{*} / z_{1}$. We have replaced $\omega$ by $\theta$ and taken the hamiltonian limit $z_{1}, z_{2}^{*} \rightarrow 0$.

Note that the limit (5.14) is the same for $r=+1$ and $r=-1$. Also, for large but finite $L$, the corrections to (5.14) vanish exponentially with $L$. The near-diagonal elements of $C_{+}$and $C_{-}$therefore become equal when $L$ is large.

Since $z_{r, m}^{L}=-r$, incrementing $i$ or $j$ in (5.13) by $L$ negates $\left(C_{r}\right)_{i, j}$ for $r=+$, and leaves it unchanged for $r=-$. This means that the near-top-right and near-bottom-left elements of $C_{+}$and $C_{-}$also tend to finite limits as $L$ becomes large, but are equal and opposite. Their sum therefore approaches exponentially to zero .

Hence when $L$ is large we expect (5.8) to become

$$
\mathcal{M}^{2}=\operatorname{det} C \text {, }
$$

where $C$ is the $L$ by $L$ matrix with elements $(i, j)$ given by (5.14). These elements tend exponentially to zero as $|i-j|$ becomes large. This is a special case of the Toeplitz matrix discussed in [4] and we can use the general result (68) therein:

$$
\lim _{L \rightarrow \infty} G^{-L} \operatorname{det} C=\exp \left(\sum_{n=1}^{\infty} n \kappa_{n} \kappa_{-n}\right)
$$


where

$$
G=\exp \left(\frac{1}{2 \pi} \int_{0}^{2 \pi} \log f(\theta) \mathrm{d} \theta\right)
$$

and

$$
\log f(\theta)=\sum_{n=-\infty}^{\infty} \kappa_{n} \mathrm{e}^{\mathrm{i} n \theta}
$$

Since $\log f(\theta)$ is an odd periodic function of $\theta$, we have $G=1$. Also, taking the logarithm of (5.15) and Laurent expanding, we readily find

$$
2 \kappa_{n}=k^{\prime n} / n \text { if } n>0,2 \kappa_{n}=-k^{-n} / n \text { if } n<0 .
$$

It follows that $\operatorname{det} C=\left(1-k^{\prime 2}\right)^{1 / 4}$, and hence

$$
\mathcal{M}=\left(1-k^{2}\right)^{1 / 8} \text {. }
$$

This is of course Onsager's famous result. [4, 3, 5].

\section{Connection with the superintegrable chi- ral Potts model}

The superintegrable case of the $N$-state chiral Potts model has some properties that closely resemble the Ising model. In particular, there is a spin-shift operator $R$ that is the natural generalization of (2.14) and divides the vector space into $N$ sub-spaces $\mathcal{V}_{Q}$, labelled by $Q=0,1, \ldots N_{1}$. Within each $\mathcal{V}_{Q}$, if one imposes the fixed-spin boundary conditions of Fig. 1, the transfer matrix $T$ generates a yet smaller sub-space of dimension $2^{m}$, where

$$
m=m_{Q}=\left[\frac{(N-1) L-Q}{N}\right] .
$$

Here $[x]$ means the integer part of the real number $x$.

Within $\mathcal{V}_{Q}, T$ is a direct product of $m$ two-by-two matrices, and there are similarity transformations that reduce the associated hamiltonian to the direct sum[11, eq.2.20]

$$
\mathcal{H}=\mu_{Q}-N \sum_{j=1}^{m}\left[\left(1-k^{\prime} \cos \theta_{j}\right) S_{j}-k^{\prime} \sin \theta_{j} C_{j}\right],
$$

where

$$
\mu=\mu_{Q}=2 k^{\prime} Q+\left(1+k^{\prime}\right)(m N-N L+L)
$$

and $\theta_{1}, \ldots \theta_{m}$ are defined by

$$
\cos \theta_{j}=\left(1+w_{j}\right) /\left(1-w_{j}\right), 0<\theta_{i}<\pi,
$$

the $w_{1}, \ldots, w_{m}$ being the zeros of

$$
P\left(z^{N}\right)=z^{-Q} \sum_{n=0}^{N-1} \omega^{(L+Q) n}\left[\left(z^{N}-1\right) /\left(z-\omega^{n}\right)\right]^{L},
$$

which is a polynomial in $w=z^{N}$ of degree $m$. 


\section{The partition functions $\tilde{Z}$}

One can explicitly calculate[12] the partition functions that generalize (4.37), (4.38):

$$
\tilde{Z}_{Q}(\alpha)=e^{-\mu \alpha} u_{1}(\alpha) \cdots u_{m}(\alpha),
$$

the function $u_{j}(\alpha)$ again being defined by (4.31), (4.32).

For $N=2$ the model reduces to the Ising model with

$$
r=1-2 Q, m(r)=m_{Q},
$$

so $Q=0$ corresponds to $R=+1$, while $Q=1$ corresponds to $R=-1$. The result (6.5) of course agrees with (4.37), (4.38), but has a slightly different form. The partition function is no longer squared on the LHS and instead of there being $L$ variables $\theta_{j}$, there are only $m$, which lie between $(L-2) / 2$ and $L / 2$. They are the same as the $\theta_{j}$ of the previous sections, but $j$ takes only the values 1 to $m$ and $0<\theta_{j}<\pi$.

These differences are easily explained. For every $\theta_{j}$ with $j=1, \ldots, m$ there is a $\theta_{j^{\prime}}=2 \pi-\theta_{j}$, where $j^{\prime}=L+1-Q-j$ and $m<j^{\prime} \leq L$. They have the same value of $u_{j}(\alpha)$ and each occurs in (4.37), (4.38), while only one occurs in (6.5). This accounts for the absence of the square in (6.5) and the presence of the factor $e^{-\mu \alpha}$ accounts for the exceptional cases $\theta_{j}=\pi$ or $2 \pi$, which are included in the product in (4.37), (4.38).

There are a total of four cases to consider.

1) $r=+1, Q=0, L=$ even: there are no exceptional cases and $m=L / 2$, $\mu=0$.

2) $r=+1, Q=0, L=$ odd: then $m=(L-1) / 2, \mu=-1-k^{\prime}$. There is an exceptional case at $j=(L+1) / 2=m+1$, where $\theta_{j}=\pi, u_{j}(\alpha)=$ $\exp \left[2\left(1+k^{\prime}\right) \alpha\right]=\mathrm{e}^{-2 \mu \alpha}$.

3) $r=-1, Q=1, L=$ even: then $m=(L-2) / 2, \mu=-2$. There are two exceptional cases at $j=L / 2$ and $L$, with $\theta_{j}=\pi$ and $2 \pi, u_{j}(\alpha)=$ $\exp \left[2\left(1+k^{\prime}\right) \alpha\right]$ and $\exp \left[2\left(1-k^{\prime}\right) \alpha\right]$, so $u_{L / 2}(\alpha) u_{L}(\alpha)=\mathrm{e}^{-2 \mu \alpha}$.

4) $r=-1, Q=1, L=$ odd: then $m=(L-1) / 2, \mu=k^{\prime}-1$. There is an exceptional case at $j=L$, with $\theta_{j}=2 \pi, u_{j}(\alpha)=\exp \left[2\left(1-k^{\prime}\right) \alpha\right]=\mathrm{e}^{-2 \mu \alpha}$.

In each case the total contribution of the exceptional cases to the RHS of (4.37) or (4.38) is $\mathrm{e}^{-2 \mu \alpha}$, which accounts for the factor $e^{-\mu \alpha}$ in (6.5).

\section{The partition function $\widetilde{W}$}

The equation (6.5) simplifies (4.37) and (4.38), expressing the $\tilde{Z}_{Q}(\alpha)$ as a product rather than the square root of a product, and reducing the number of factors from $L$ to $m$ or $m^{\prime}$, where

$$
m=m(+)=[L / 2], m^{\prime}=m(-)=[(L-1) / 2] .
$$

Can we similarly reduce the expression (4.57) for $\widetilde{W}(\alpha, \beta, x)$ ?

The answer is yes, apart from simple factors that are independent of $x$ and easily calculated. We show that the matrix

$$
G=\tilde{U}_{+}(\alpha) F_{+} \tilde{U}_{-}(\beta)+x \tilde{V}_{+}(\alpha) F_{+} \tilde{V}_{-}(\beta)
$$


can be reduced to block lower-triangular form and its determinant expressed as simple factors times the square of an $m^{\prime}$ by $m^{\prime}$ determinant.

For brevity, in the following two sub-sections, unless indicated otherwise we write

$$
\begin{gathered}
\theta_{j}=\theta_{+, j}, \quad \theta_{j}^{\prime}=\theta_{-, j}, \\
u_{j}=\tilde{U}_{+}(\alpha)_{j, j}=u_{+, j}(\alpha), \quad v_{j}=\tilde{V}_{+}(\alpha)_{j, j}=v_{+, j}(\alpha), \\
u_{j}^{\prime}=\tilde{U}_{-}(\beta)_{j, j}=u_{-, j}(\beta), \quad v_{j}^{\prime}=\tilde{V}_{-}(\beta)_{j, j}=v_{-, j}(\beta) .
\end{gathered}
$$

We use the definition (4.51) of $F_{ \pm}$and the properties (4.52), (6.12). We also implicitly use

$$
u_{L+1-j}=u_{j}, v_{L+1-j}=-v_{j}, u_{L-j}^{\prime}=u_{j}^{\prime}, v_{L-j}^{\prime}=-v_{j}^{\prime} .
$$

We do not in this section need the definitions (4.31).

\section{The case $L$ even}

In this case note that the $\tilde{U}_{+}(\alpha), \tilde{V}_{+}(\alpha)$ on the left of (6.8) depend on the $\theta_{j}$, which satisfy $\theta_{i}+\theta_{L+1-i}=2 \pi$, so it is natural to combine rows $i$ and $L+1-i$. The $\tilde{U}_{-}(\beta), \tilde{V}_{-}(\beta)$ on the right involve $\theta_{j}^{\prime}$, satisfying $\theta_{j}^{\prime}+\theta_{L-j}^{\prime}=2 \pi$, so it is natural to combine columns $j$ and $L-j$. Also, columns $L / 2$ and $L$ both correspond to exceptional values of $\theta_{j}^{\prime}$, where $\sin \theta_{j}^{\prime}=0$. The elements $v_{L / 2}^{\prime}, v_{L}^{\prime}$ of $\tilde{V}_{-}(\beta)$ vanish for these two columns. From (6.7),

$$
m=L / 2, m^{\prime}=(L-2) / 2=m-1 .
$$

We perform the following equivalence transformations sequentially on $G$ :

$$
\begin{array}{llll}
\text { 1) } & G_{i j} \rightarrow & G_{i j}+G_{L+1-i, j}, & 1 \leq i \leq m, 1 \leq j \leq L \\
\text { 2) } G_{i j} \rightarrow & G_{i j}+G_{i, L-j}, & m<j<L \\
\text { 3) } G_{i, L} \rightarrow \quad G_{i, L}-u_{L}^{\prime} G_{i, m} / u_{m}^{\prime}, & \\
\text { 4) } G_{i, j} \rightarrow \quad G_{i, j}-u_{j}^{\prime} G_{i, m} / u_{m}^{\prime}, & 1 \leq j<m \\
\text { 5) } G_{i, j} \rightarrow & G_{i, j}-2 u_{j}^{\prime} G_{i, m} / u_{m}^{\prime}, & m<j<L \\
\text { 6) } G_{i, j} \rightarrow & G_{i, j}-\left(1+c_{j}^{\prime}\right) u_{j}^{\prime} G_{i, L} / u_{L}^{\prime}, & m<j<L
\end{array}
$$

where $c_{j}^{\prime}=\cos \left(\theta_{j}^{\prime}\right)$.

The first transformation corresponds to pre-multiplying $G$ by some matrix, all the others to post-multiplying it. Steps (2) to (6) are for all values of $i$, i.e. $1 \leq i \leq L$. At every step we are merely incrementing rows or columns by linear combinations of other rows or columns, so the determinant of $G$ is unchanged.

The elements $F_{i, j}$ of $F_{+}$satisfy

$$
F_{i j}+F_{L+1-i, L-j}=F_{i, L}+F_{L+1-i, L}=-2 / L
$$

for all $i, j$. It follows that the final matrix $G$ is such that

$$
G_{i, j}=0 \text { for } 1 \leq i \leq m, m<j \leq L .
$$


Thus $G$ is now block lower-triangular:

$$
G=\left(\begin{array}{ll}
Y & 0 \\
\mathcal{T} & \mathcal{Z}
\end{array}\right)
$$

where the $Y, \mathcal{T}, \mathcal{Z}$ are $m$ by $m$ matrices. We find

$$
\begin{gathered}
Y_{i, j}=\frac{-21\left(\sin \theta_{j}^{\prime} u_{i} u_{j}^{\prime}+x \sin \theta_{i} v_{i} v_{j}^{\prime}\right)}{L\left(\cos \theta_{i}-\cos \theta_{j}^{\prime}\right)} \\
Y_{i, m}=-2 u_{i} u_{m}^{\prime} / L
\end{gathered}
$$

for $1 \leq i \leq m, 1 \leq j<m$.

Note that the equivalence transformations are independent of $x$, so $Y, \mathcal{T}, \mathcal{Z}$ are all linear in $x$, their coefficients being the transforms of the corresponding coefficients of $G$.

We define the $m$ by $m$ matrix $\mathcal{Z}$ by (6.14), but with the rows and columns re-arranged so that, for $1 \leq i, j \leq m$,

$$
(\mathcal{Z})_{i, j}=(G)_{L+1-i, j^{\prime}}
$$

where $j^{\prime}=L$ if $j=m$, else $j^{\prime}=L-j$. Let $D, D^{\prime}$ be the diagonal matrices with elements

$$
D_{j, j}=\sin \theta_{i}, \quad D_{j, j}^{\prime}=\sin \theta_{i}^{\prime}
$$

for $j=1, \ldots, m$, except that $D_{m, m}^{\prime}=-1 u_{L}^{\prime} / u_{m}^{\prime}$. Then we find that

$$
\mathcal{Z}=-D^{-1} Y D^{\prime}
$$

Hence

$$
\operatorname{det} G=\operatorname{det}\left(-D^{\prime}\right)(\operatorname{det} Y)^{2} / \operatorname{det} D
$$

and we see that $\operatorname{det} G$ is basically the square of the $m$ by $m$ determinant $\operatorname{det} Y$.

We can do better yet and write it in terms of the square of an $m^{\prime}=m-1$ determinant. Expand the matrices $G, Y$ in powers of $x$ :

$$
G=\mathbf{g}_{0}+x \mathbf{g}_{1}, Y=\mathbf{y}_{0}+x \mathbf{y}_{1}
$$

so $\mathbf{g}_{0}, \mathbf{g}_{1}, \mathbf{y}_{0}, \mathbf{y}_{0}$ are $x$-independent matrices and $\mathbf{g}_{0}=\tilde{U}_{+}(\alpha) F_{+} \tilde{U}_{-}(\beta)$. From (4.52), its inverse is

$$
\mathbf{g}_{0}^{-1}=\tilde{U}_{-}(\beta)^{-1} F_{-} \tilde{U}_{+}(\alpha)^{-1} .
$$

We can follow the above six steps (6.11) and evaluate $\mathbf{g}_{0}^{-1}$ after the six equivalence transformations. It also becomes block lower-triangular, as in (6.14). In particular its top-left block is $\mathbf{y}_{0}^{-1}$, with elements

$$
\begin{gathered}
{\left[\mathbf{y}_{0}^{-1}\right]_{i, j}=\frac{-21 \sin \theta_{i}^{\prime}}{L\left(\cos \theta_{i}^{\prime}-\cos \theta_{j}\right) u_{i}^{\prime} u_{j}},} \\
{\left[\mathbf{y}_{0}^{-1}\right]_{m, j}=-1 /\left(u_{m}^{\prime} u_{j}\right),}
\end{gathered}
$$


for $1 \leq i<m, 1 \leq j \leq m$. This matrix is necessarily the inverse of $\mathbf{y}_{0}$ and from 6.19

$$
\operatorname{det} \mathbf{g}_{0}^{-1} G=\left[\operatorname{det} \mathbf{y}_{0}^{-1} Y\right]^{2} .
$$

We calculate $\mathbf{y}_{0}^{-1} Y$. From (6.15), the last column of $\mathbf{y}_{1}$ vanishes. Hence, using (6.20),

$$
\mathbf{y}_{0}^{-1} Y=I+x \tilde{\mathcal{D}}
$$

where $\tilde{\mathcal{D}}=\mathbf{y}_{0}^{-1} \mathbf{y}_{1}$ and again the last column of $\mathcal{D}$ vanishes. The determinant of the RHS is therefore the same as that of the RHS truncated to its first $m^{\prime}=m-1$ rows and columns.

We can therefore ignore the last column of $Y$ and the last row of $\mathbf{y}_{0}^{-1}$ when calculating the LHS, and contract $Y$ to the non-square $m$ by $m^{\prime}$ matrix with elements given by the first of the equations (6.15), $\mathbf{y}_{0}^{-1}$ to the $m^{\prime}$ by $m$ matrix with elements given by the first of the equations (6.22).

We define the $m$ by $m^{\prime}$ matrix $A_{+}$and the $m^{\prime}$ by $m$ matrix $A_{-}$by

$$
\left(A_{+}\right)_{i, j}=\frac{2 \sin \theta_{i}}{L\left(\cos \theta_{i}-\cos \theta_{j}^{\prime}\right)}, \quad\left(A_{-}\right)_{i, j}=\frac{2 \sin \theta_{i}^{\prime}}{L\left(\cos \theta_{i}^{\prime}-\cos \theta_{j}\right)}
$$

Then from (6.15), (6.22),

$$
\mathbf{y}_{1}=-i \tilde{V}_{+}(\alpha) A_{+} \tilde{V}_{-}(\beta), \mathbf{y}_{0}^{-1}=-{ }_{1} \tilde{U}_{-}(\beta)^{-1} A_{-} \tilde{U}_{+}(\alpha)^{-1},
$$

where we now take the $\tilde{U}_{r}, \tilde{V}_{r}, \tilde{X}_{r}$ to be the diagonal matrices defined by (4.31), (4.58), but truncated to the first $m(r)$ terms.

Hence

$$
\tilde{\mathcal{D}}=-\tilde{U}_{-}(\beta)^{-1} \tilde{D}_{-}(\beta)
$$

where

$$
\mathcal{D}=-A_{-} X_{+}(\alpha) A_{+} X_{-}(\beta) .
$$

From (6.23), we obtain

$$
\operatorname{det} G=\operatorname{det}\left(\tilde{U}_{+}(\alpha) F_{+} \tilde{U}_{-}(\beta)\right)\left[\operatorname{det}\left(I_{m^{\prime}}+x \mathcal{D}\right)\right]^{2},
$$

where $I_{m^{\prime}}$ is the identity $m^{\prime}$ by $m^{\prime}$ matrix.

The relations (4.57), (4.59) therefore become

$$
\widetilde{W}(\alpha, \beta, x)=\tilde{Z}_{+}(\alpha) \tilde{Z}_{-}(\beta) \operatorname{det}\left[I_{m^{\prime}}-x A_{-} X_{+}(\alpha) A_{+} X_{-}(\beta)\right] .
$$

We have eliminated the square on the LHS and used only matrices of dimensions $m$ or $m^{\prime}$, involving only $\theta_{1}, \ldots, \theta_{m}, \theta_{1}^{\prime}, \ldots, \theta_{m^{\prime}}^{\prime}$.

\section{The case $r=+, L$ odd}

Now (6.7) gives $m=m^{\prime}=(L-1) / 2$, so the matrix $G$ has one exceptional row at $i=m+1$ and one exceptional column at $j=L$. The steps corresponding to (6.11) are

$$
\begin{array}{ccc}
\text { 1) } G_{i j} \rightarrow \quad G_{i j}+G_{L+1-i, j}, & & 1 \leq i \leq m, 1 \leq j \leq L \\
\text { 2) } G_{i j} \rightarrow G_{i j}-2 u_{i} G_{m+1, j} / u_{m+1}, & 1 \leq i \leq m, 1 \leq j \leq L
\end{array}
$$



3) $G_{i j} \rightarrow$
$G_{i j}+G_{i, L-j}$,
$m<j<L$
4) $G_{i, j} \rightarrow G_{i, j}-2 u_{j}^{\prime} G_{i, L} / u_{L}^{\prime}$
$m<j<L$
5) $G_{i, j} \rightarrow$
$G_{i, j}-u_{j}^{\prime} G_{i, L} / u_{L}^{\prime}$
$1 \leq j \leq m$

The first two transformations correspond to pre-multiplying $G_{r}$ by some matrix, all the others to post-multiplying it. Steps (3) to (6) are for all values of $1 \leq i \leq L$

Using (6.12), we find that the transformed $G$ has the structure

$$
G=\left(\begin{array}{ccc}
Y & 0 & 0 \\
. \cdot & 0 & -u_{m+1} u_{L}^{\prime} \\
\mathcal{T} & \mathcal{Z} & . \cdot
\end{array}\right)
$$

where $Y, \mathcal{T}, \mathcal{Z}$ are all $m$ by $m^{\prime}=m$ blocks and the middle row and last column have width one. Hence

$$
\operatorname{det} G=u_{m+1} u_{L}^{\prime}(\operatorname{det} Y)(\operatorname{det} \mathcal{Z}) .
$$

we also find

$$
Y_{i j}=\frac{-41 \cos ^{2}\left(\theta_{i} / 2\right)\left[u_{i} u_{j}^{\prime} \tan \left(\theta_{j}^{\prime} / 2\right)+x v_{i} v_{j}^{\prime} \tan \left(\theta_{i} / 2\right)\right]}{L\left(\cos \theta_{i}-\cos \theta_{j}^{\prime}\right)},
$$

and again if we rearrange $\mathcal{Z}$ so that $\mathcal{Z}_{i j}=G_{L+1-i, L-j}$ for $1 \leq i, j \leq m$, then

$$
\mathcal{Z}=-D^{-1} Y D^{\prime}
$$

with $D, D^{\prime}$ given by (6.17) for all $1 \leq j \leq m$. Further,

$$
\left(\mathbf{y}_{0}^{-1}\right)_{i j}=\frac{-21 \sin \theta_{i}^{\prime}}{L\left(\cos \theta_{i}^{\prime}-\cos \theta_{j}\right) u_{i}^{\prime} u_{j}} .
$$

From (6.15) and (6.32), $\mathbf{y}_{0}$ is different for the two cases ( $L$ even and $L$ odd). However, the wanted elements of $\mathbf{y}_{1}$ and of $\mathbf{y}_{0}^{-1}$ are the same, so again $\widetilde{W}(\alpha, \beta, x)$ is given by (6.29), with $A_{+}, A_{-}$given by (6.25).

From (4.31), (4.58), the diagonal matrices $X_{ \pm}$have diagonal elements

$$
\begin{aligned}
{\left[X_{+}(\alpha)\right]_{j j} } & =-\frac{k^{\prime} \sin \theta_{j} \sinh \left(2 \alpha \lambda_{j}\right)}{\lambda_{j} \cosh \left(2 \alpha \lambda_{j}\right)+\left(1-k^{\prime} \cos \theta_{j}\right) \sinh \left(2 \alpha \lambda_{j}\right)} \\
{\left[X_{-}(\beta)\right]_{j j} } & =-\frac{k^{\prime} \sin \theta_{j}^{\prime} \sinh \left(2 \beta \lambda_{j}^{\prime}\right)}{\lambda_{j}^{\prime} \cosh \left(2 \beta \lambda_{j}^{\prime}\right)+\left(1-k^{\prime} \cos \theta_{j}^{\prime}\right) \sinh \left(2 \beta \lambda_{j}^{\prime}\right)},
\end{aligned}
$$

$\lambda_{j}$ being defined by (4.32) and $\lambda_{j}^{\prime}$ by the same equation with $\theta_{j}$ replaced by $\theta_{j}^{\prime}$. 


\section{$7 \widetilde{W}$ in terms of orthogonal matrices $B_{+}, B_{-}$}

In this section we take the result (6.29) and write it in terms, not of the matrices $A_{+}, A_{-}$, but of related orthogonal matrices $B_{+}, B_{-}$.

In both the $L$ even and odd cases, the matrices $\mathbf{y}_{0}^{-1}, \mathbf{y}_{0}^{T}$ above have a very similar structure, differing only by diagonal equivalence transformations. We can therefore construct orthogonal matrices $B_{+}, B_{-}$that differ from $A_{+}, A_{-}$ only by such diagonal equivalence transformations.

Let $E, E^{\prime}$ be diagonal matrices with diagonal elements

$$
\begin{array}{ll}
E_{j j}=\sin \theta_{j}, E_{j j}^{\prime}=\sin \theta_{j}^{\prime} & \text { if } L=\text { even }, \\
E_{j j}=\tan \left(\theta_{j} / 2\right), E_{j j}^{\prime}=\tan \left(\theta_{j}^{\prime} / 2\right) & \text { if } L=\text { odd } .
\end{array}
$$

Then whether $L$ is even or odd, we find from (6.15), (6.17), (6.22), (6.25), (6.32), (6.34) that

$$
\mathbf{y}_{0}^{-1}={ }_{1} D^{\prime} A_{+}^{T} D^{-1}, \mathbf{y}_{0}=-1 E^{-1} A_{+} E^{\prime}, A_{-}^{T}=-D^{-1} A_{+} D^{\prime},
$$

$\mathbf{y}_{0}^{-1}$ being the left-inverse of $\mathbf{y}_{0}$.

Hence we can define matrices $B_{+}, B_{-}$:

$$
B_{+}=(D E)^{-1 / 2} A_{+}\left(D^{\prime} E^{\prime}\right)^{1 / 2} \quad, \quad B_{-}=\left(E^{\prime} / D^{\prime}\right)^{1 / 2} A_{-}(D / E)^{1 / 2}
$$

such that

$$
B_{-} B_{-}^{T}=B_{+}^{T} B_{+}=I_{m^{\prime}}, B_{-}=-B_{+}^{T},
$$

so, even though they are not necessarily square, they are orthogonal in this sense. Their elements are

$$
\begin{aligned}
& \left(B_{+}\right)_{i j}=-\left(B_{-}\right)_{j i}=\frac{2 \sin \theta_{j}^{\prime}}{L\left(\cos \theta_{i}-\cos \theta_{j}^{\prime}\right)} \quad \text { if } L=\text { even } \\
& \left(B_{+}\right)_{i j}=-\left(B_{-}\right)_{j i}=\frac{4 \cos \left(\theta_{i} / 2\right) \sin \left(\theta_{j}^{\prime} / 2\right)}{L\left(\cos \theta_{i}-\cos \theta_{j}^{\prime}\right)} \text { if } L=\text { odd } .
\end{aligned}
$$

Set

$$
E_{+}=E, E_{-}=E^{-1} .
$$

then we can write the result (6.29) as

$$
\widetilde{W}(\alpha, \beta, x)=\tilde{Z}_{+}(\alpha) \tilde{Z}_{-}(\beta) \operatorname{det}\left[I_{m^{\prime}}-x X_{+}(\alpha) E_{+} B_{+} X_{-}(\beta) E_{-} B_{-}\right] .
$$

We can slightly generalize this result. Write $\widetilde{W}(\alpha, \beta, x)$ as $\widetilde{W}_{+}(\alpha, \beta, x)$, and define a new function $\widetilde{W}_{-}(\alpha, \beta, x)$ to be given by (3.6) with $v_{+}, v_{-}$interchanged. Thus (3.5), (3.6) become

$$
\tilde{Z}_{r}(\alpha)=v_{r}^{\dagger} \mathrm{e}^{\alpha \mathcal{H}} v_{r}, \widetilde{W}_{r}(\alpha, \beta, x)=v_{r}^{\dagger} \mathrm{e}^{\alpha \mathcal{H}} \mathrm{e}^{\rho \mathcal{J}} S_{1} \mathrm{e}^{\beta \mathcal{H}} v_{-r} .
$$

Then we can generalize (7.7) to

$$
\widetilde{W}_{r}(\alpha, \beta, x)=\tilde{Z}_{r}(\alpha) \tilde{Z}_{-r}(\beta) \operatorname{det}\left[I-x X_{r}(\alpha) E_{r} B_{r} X_{-r}(\beta) E_{-r} B_{-r}\right] \text {, }
$$

for $r= \pm$, where $I$ is the identity matrix of dimension $m(r)$. For $r=+$, (7.9) is (7.7). For $r=-$ it can be deduced from it by taking the hermitian conjugate of the definition (7.8) of $\widetilde{W}_{-}$and interchanging $\alpha$ with $\beta$. We also need the general identity

$$
\operatorname{det}(I+A B)=\operatorname{det}(I+B A),
$$

which is true for all matrices $A, B$ even when they are non-square, 


\section{Conclusions}

We have considered the zero-field Ising model on a cylindrical lattice with fixed spin boundary condtions on the top and bottom rows, and have replaced the transfer matrix product by the exponential of the associated hamiltonian. This leaves the eigenvectors and spontaneous magnetization unchanged.

We have then used the Clifford algebra technique of Kaufman[2] to evaluate as $L$ by $L$ determinants the partition function $\tilde{Z}$, and the partition function $\widetilde{W}$ with a single-spin operator $S_{1}$ included. In section 5 we show how to use Szegö's theorem on Toeplitz matrices to then obtain the spontaneous magnetization.

Much of this merely parallels Yang's derivation [5], but in section 6 we go on to reduce $\tilde{Z}$ and $\widetilde{W}$ to determinants of size $m$ or $m^{\prime}$ where $L-2 \leq 2 m \leq L$.

In this form we can compare the Ising model results for $\tilde{Z}$ with those of the $N$-state superintegrable chiral Potts model, taking the hamiltonian limit therein. [11, 12] The Ising model is the superintegrable chiral Potts model with $N=2$, and the results of course agree.

All this leads up to the question whether we can generalize the Ising result (6.29) or (7.9) for $\widetilde{W}$ to the $N$-state superintegrable chiral Potts model, for general $N$. This should open up the possibility of an algebraic derivation of the spontaneous magnetization of that model. We have numerical evidence that strongly suggests the answer to the question is yes, and that one can make a fairly immediate generalization of the form of the result given in (7.9) in terms of the orthogonal matrices $B_{+}, B_{-}$. We shall present the conjecture in an accompanying paper.[12]

\section{References}

[1] Onsager, L.: Crystal statistics. I. A two-dimensional model with an order-disorder transition. Phys. Rev 65, 117-149 (1944)

[2] Kaufman, B.: Crystal statistics. II. Partition function evaluated by spinor analysis. Phys. Rev 76, 1232-1243 (1949)

[3] Onsager, L. In: Proceedings of the IUPAP conference on statistical mechanics, "Discussione e observazioni", Nuovo Cimento (Suppl), Series 9, 6, 261 (1949)

[4] Montroll, E. W., Potts, R. B., Ward, J. C.: Correlations and spontaneous magnetization of the two-dimensional Ising model. J. Math. Phys. 4, 308-322 (1963)

[5] Yang, C. N.: The spontaneous magnetization of a two-dimensional Ising model. Phys. Rev. 85, 808-816 (1952)

[6] Baxter, R. J.: Derivation of the order parameter of the chiral Potts model. Phys. Rev. Lett. 94, 130602 (2005)

[7] Baxter, R. J.: The order parameter of the chiral Potts model. J. Stat. Phys. 120, 1-36 (2005)

[8] Grenander, U., Szegö, G.: Toeplitz forms and their applications. Univ. Calif. Press, Berkeley, (1958) 
[9] Baxter, R. J.: Exactly solved models in statistical mechanics. Academic Press, London/San Diego1989; Dover Publications, Mineola, NY (2007)

[10] Baxter, R. J.: Corner transfer matrices of the eight-vertex model. II. The Ising model case. J. Stat. Phys. 17, 1-14 (1977)

[11] Baxter, R. J.: Superintegrable chiral Potts model: thermodynamic properties, an "inverse" model, and a simple associated hamiltonian. J. Stat. Phys. 57, 1-39 (1989)

[12] Baxter, R. J.: A conjecture for the superintegrable chiral Potts model. J. Stat. Phys. 132, ??-?? (2008) 